\title{
Porch Design Based on Family Health Needs
}

\author{
Yongbo Liu* \\ Suan Sunandha Rajabhat University, Bangkok, Thailand \\ *Corresponding author: Yongbo Liu, 935389147@qq.com
}

Copyright: (C) 2022 Author(s). This is an open-access article distributed under the terms of the Creative Commons Attribution License (CC BY 4.0), permitting distribution and reproduction in any medium, provided the original work is cited.

\begin{abstract}
Physical health is the basic need for survival. There are many factors affecting health, and pathogenic bacteria and viruses are the main reasons. A healthy lifestyle can effectively reduce the risk of disease, and family living space is the main place of life, so improving family health awareness and living habits is an effective measure to maintain health. Indoor living space and outdoor space are relatively independent. The porch is the entrance of the family and the buffer space connecting indoor and outdoor. Therefore, optimizing the sanitary design of the porch is not only conducive to blocking the spread of viruses and bacteria, but also to form a good health awareness and maintain healthy living habits.
\end{abstract}

Keywords: Health needs; Porch design; Functionality; Health awareness

Online publication: January 18, 2022

\section{Introduction}

Health is people's hope for their own existence, and people link health and happiness together, and link health and social progress together ${ }^{[1]}$. With the continuous progress of the times and the sustainable development of the economy, people's problem with food and clothing has been fundamentally changed, and life expectancy is also gradually increasing. However, the problem of physical health is still a major problem perplexing people's life. With the progress of medicine, pathogenic bacteria and viruses are also evolving, and even drug resistance appears. General diseases can be cured or relieved by drugs, which cannot arouse people's strong awareness of paying attention to hygiene. However, in the early twenty-first century, the world was attacked repeatedly by deadly viruses, such as SARS, avian flu, Ebola and etc. Especially the deadly infectious epidemic outbreak in 2020, which made people realize the importance of virus and health. Therefore, in order to maintain physical fitness, preventing the invasion of bacteria and viruses is the key.

Home space is an important place for family life and close communication, which is prone to cross infection, especially the way of living in modern urban areas. With dense personnel and serious air and water pollution, it is very important to set disinfection facilities at the entrance to the indoor space in order to prevent the indoor spread of outdoor bacteria and viruses. At this time, it highlights the importance and necessity of porch design. The research object of this paper is the living space of urban communities in China. It is mainly aimed at designing and studying the basic functions and epidemic prevention and disinfection functions of the porch, and carrying out the functional layout of the porch cabinet according to the structure and behavior habits of family members. So as to imperceptibly improve the behavior and habits of family members, form a good health awareness and meet the health needs of family members. 


\section{The basic and new functions of the porch}

The concept of porch comes from the "screen wall" in traditional Chinese architecture. However, the Japanese were the first to position the porch as a functional space in interior design ${ }^{[2]}$. The porch, also known as the entrance hall, is mainly used to place the items that need to be replaced in and out of the house, as well as the starting point and end point for welcoming and sending guests. The application of porch is more and more extensive, and has become an indispensable functional space for home design. Under the new health needs, the functionality of the porch should also be constantly updated and changed from time to time.

\subsection{Basic functions of the porch}

\subsubsection{Guide streamline}

The porch space is a hub area with the function of passage and for entering the interior from the outside and stepping into other interior spaces. It includes the passage of people and the entry and exit of goods, which all need to go through the porch, or even is the only channel. The indoor traffic flow line will be designed according to people's behavior habits, ergonomics and psychology, and the reasonable functional space will be arranged reasonably, all of which take the porch as the starting point.

\subsubsection{Cleaning and pollution zoning}

Outdoor sediment, dust, oil, harmful bacteria and viruses may attach to clothes, shoes, personal belongings or skin, so the cleaning function of the porch is very important for a healthy life. The clean and dirty partition of the porch takes the porch space as the "polluted area" and other indoor spaces as the "clean area." The reasonable design of the porch can prevent most of the outdoor pollutants from being brought into other indoor spaces.

\subsubsection{Thermal insulation}

The design of the thermal insulation function of the entrance can be traced back to ancient times. A bonfire or stove pit is set at the entrance of the living cave to heat the air entering the cave and then circulate to the living area to keep warm. The indoor porch of modern residence is different from the original thermal insulation method, but the concept of improving indoor temperature is the same. The current function is mainly to keep warm, avoid strong hedging of indoor and outdoor cold and warm air, and play a buffer role.

\subsubsection{Obscuring sight}

Whether it is traditional Chinese architecture or modern interior design, the porch has the function of blocking the line of sight, avoiding the unobstructed view of the interior space when the door is pushed in. Psychologically, it has a certain role in protecting privacy, so that newcomers can maintain a comfortable sense of distance from indoor members, which is also in line with the implicit and obscure traditional thought of the Chinese people. It is generally divided into full occlusion and semi occlusion.

\subsubsection{Changing shoes and clothes}

Changing shoes at the porch mainly refers to: changing shoes suitable for outdoor wear when going out, and changing loose and comfortable shoes when entering the room; trapping outdoor dirt at the porch to protect the cleanliness of other indoor spaces, and sometimes even distinguishing different shoes between family members and guests. The role of changing clothes mainly refers to: when you go out, take off your coat and when you enter the room wash it, or change your home clothes suitable for indoor wear, which also prevent the dirty coat from spreading to more indoor space. 


\subsubsection{External exchange}

Home space is the family's internal activity space, and has a certain social communication function. For example, the visits of relatives or friends, as well as the reception of online shopping and takeout in modern life, all need to contact the outside world through the porch. It can also play the role of internal and external spacing to avoid too many unnecessary people or things disturbing indoor life, such as some door-to-door sales and neighborhood consultation.

\subsubsection{Storage function}

The area of the porch is limited, but there are many kinds of items to be placed and stored, such as bags, clothes, shoes, umbrellas, keys, etc. Porch cabinets are often used as storage tools. According to the use frequency and season, it is divided into high and low cabinets and large and small storage grids. Reasonable zoning can ensure convenient use.

\subsection{New functions of the porch}

The basic function of the porch can only meet the basic use function. Under the new health needs, the new function of the porch is to have the killing function, that is, the role of disinfection and sterilization. It is necessary to increase the functional zoning of the porch and disinfection and sterilization facilities, such as the placement of wash basins, disinfectants, masks and shoe covers, as well as shoe cabinets equipped with ultraviolet lights. The virus is sensitive to ultraviolet and heat. Lipid solvents such as ether, $75 \%$ ethanol, chlorine disinfection, peracetic acid, and chloroform at $56^{\circ} \mathrm{C}$ for 30 minutes, can inactivate the virus, however chlorhexidine cannot ${ }^{[3]}$. When individuals enter the room, the surfaces of all things that come into touch with the outside world should be cleansed and disinfected, primarily by changing clothing and shoes, washing hands and faces, and etc. It is required to establish suitable functional zoning and usage sequence in the porch cabinet for the protection goods against general germs and viruses. As a result, the design of the porch cabinet should be based on the usage requirements of various family members.

\section{Family members' needs for the use of the porch}

Family is the cell of society. It is a social life organization produced by marriage, kinship or adoption. It is the closest social group ${ }^{[4]}$. Home space generally refers to the living space of family members. The porch is an important part of the spacing space. The design of the porch only needs to meet the residents' preferences and habits, and does not need to consider the needs of others too much. However, it also has some commonalities, because the way of human life is similar. Although the porch design is affected by region, climate, age, occupation, gender and culture, the requirements for the basic functions and killing functions of the porch are the same. Therefore, according to different age groups and taking entering and leaving the porch as research conditions, we can analyze the similar living laws and needs of different people for the porch.

\subsection{Minors (0-17 years old)}

Minors need the care of their families before school age, with relatively simple and small size supplies. In school age, their main task is to study, and their supplies are generally related to learning and sports. The general rule of their life is to put a schoolbag on the back of breakfast, change clothes and shoes, and go out with keys and school cards. After school, they take toys or balls home after outdoor sports, or go home directly, put their belongings, umbrellas, raincoats, etc., take off their hat and mask, take off their coat, put on their home clothes and shoes, and then wash their hands before entering the indoor living area. 


\subsection{Youth and middle age (18-59 years old)}

The lives of young and middle-aged people are relatively diversified, and they need to deal with complex family affairs and social activities, mainly related to work, communication and taking care of their families. In the porch, the use frequency is higher and the demand is more. The general rule of their life is to put on work clothes or decent clothes in the morning, sit on the shoe changing stool, put on leather shoes or high heels, dress in the changing mirror, and go out with a satchel or backpack, as well as car keys, access control cards, etc. After work, they either take their own sports goods to exercise outdoors, or put things at home, put on home clothes, take off shoes, hats and masks, wash their hands and enter the living area. If relatives and friends visit, they can change home clothes in the hallway, wear shoe covers, wash their hands after disinfection and go into the living area.

\subsection{Elderly (over 60 years old)}

The life of the elderly is generally dominated by retirement, and they will also participate in some social activities. Their living habits are not different from those of other adults. In the use of the porch, it is necessary to add some designs that care for the elderly, such as increasing the storage space of crutches or wheelchairs, lowering the height of shoe changing stools, and making anti-skid treatment on the ground. The general rule of their life is to get up in the morning, put on comfortable clothes and shoes, take props or equipment to outdoor morning exercise, then go to the vegetable market to buy vegetables and go home, put crutches and handy items, change shoes, wash hands and disinfect before entering the living area. In the afternoon, they change into more formal clothes and shoes, hold keys, access cards, satchels or some props, and find friends to drink tea, chat or talent training. At night, they go home, put umbrellas, crutches or wheelchairs and other tools, wash hands, disinfect and change into household clothes before entering the living area.

\section{Functional layout of the porch}

The porch is not only the conversion space inside and outside the house, but also carries rich use functions, which provide convenience for people's lives. At the same time, the reasonable porch design makes people more cheerful on emotion ${ }^{[5]}$. The porch design should not only meet the basic needs of family life, but also carry out the porch cabinet layout according to the living habits of family members. On this basis, add a new killing function layout, so as to meet the requirements of health and hygiene in the new era. The basic functions do not need to be discussed repeatedly, and the new functional layout needs to be designed and discussed. It is mainly designed from the layout of the cleaning partition of the plane layout, the layout form of the porch cabinet, and the location of the articles.

\subsection{Cleaning and pollution zoning of the porch}

Currently, the porch must simply distinguish between dirty and clean areas in order to fulfil basic sanitary requirements, but its cleaning capability is severely restricted. The new porch design includes disinfection and sterilization features, which may split the porch space into dirty, semi-dirty, and clean areas based on the order in which people enter the entrance. Subdivision is used to fine-tune the problem, better organize cleaning and disinfection orders, and better organize the storage of items. If the indoor area is large enough, a separate porch room can be created with sliding doors to divide it from the rest of the house.

\subsection{Layout of the porch}

The size of the porch is mainly affected by the architectural designer and design requirements. The porch will be divided according to the size and orientation of the indoor area. The layout of the porch is determined according to the preferences of users and the number of family members. The forms of the porch are mainly 
distinguished according to the design of the porch cabinet. The current porch design forms are roughly divided into three types: “一” word style, “L" model and “凹” word style. The three forms are mainly different in size and similar or the same in function.

\subsection{Functional zoning of porch cabinet}

The function of the porch is reflected in the regional division and the design of the porch cabinet. Its cleaning function and storage function need the cabinet as the carrier. According to people's behavior and habits, the use of ergonomics, the cabinet is divided into partitions, to maximize the use of space and improve convenience. Partition storage is to divide the storage space into small areas and place the stored items in different areas according to certain rules ${ }^{[6]}$. Generally, some articles that are not suitable for entering the room too much are placed near the entrance, such as umbrellas, raincoats and shoe covers. Commonly used shoes, keys, coats and other items are placed in a readily accessible position for easy access. Infrequently used seasonal goods and entertainment equipment are stored in high places or corners (Table 1).

Table 1. Functional zoning of porch cabinet

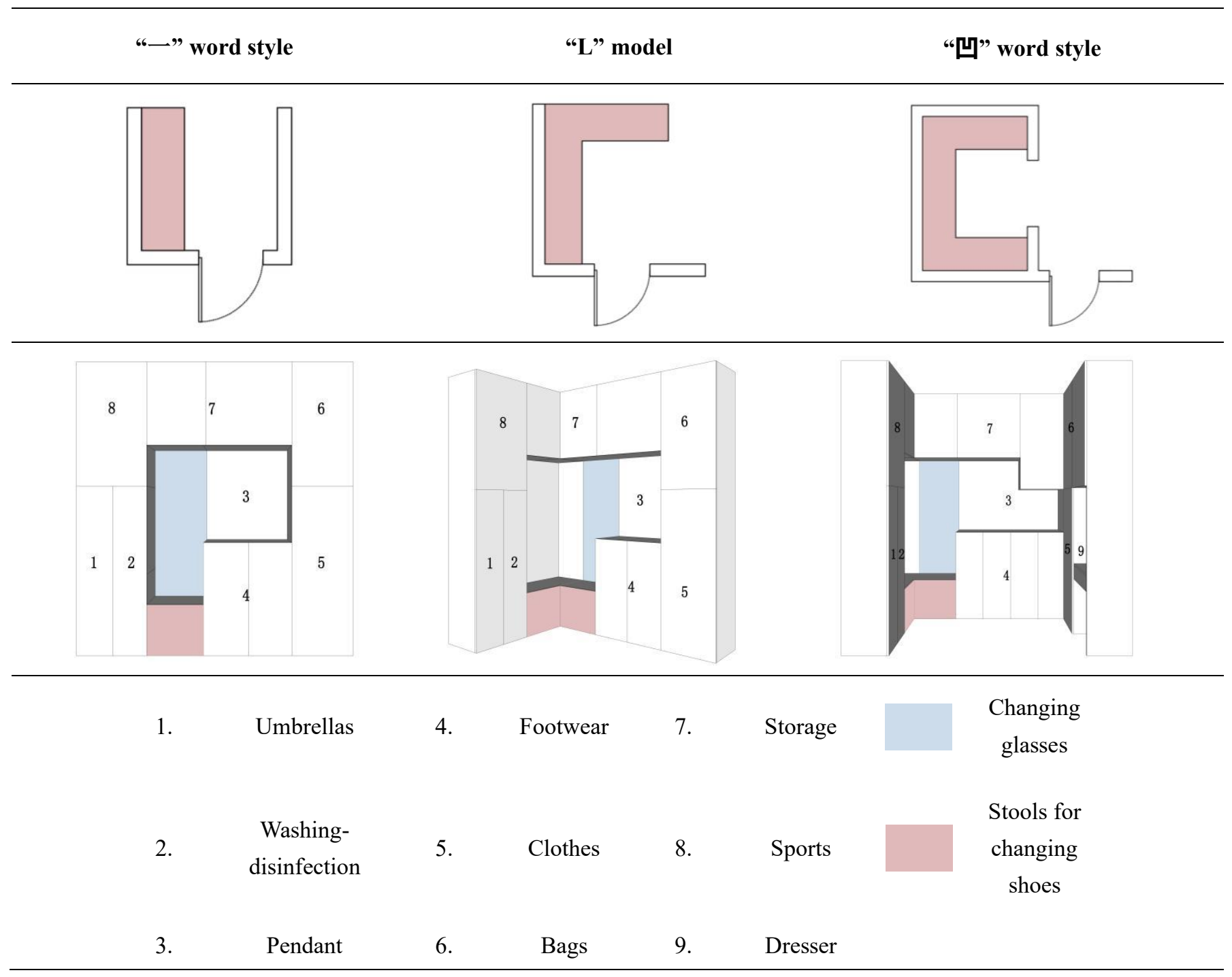



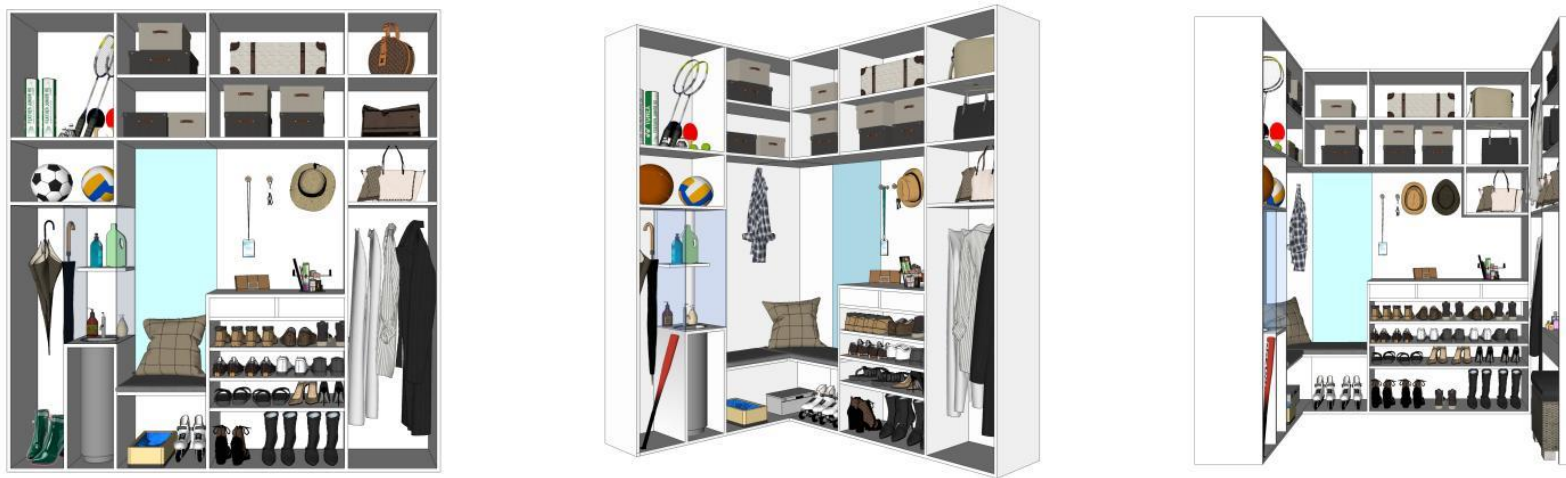

Source of the form: Self-made according to data

\section{Conclusion}

Everyone requires a healthy body to live, and health is the foundation of survival. Physical well-being requires increased health awareness and the development of healthy lifestyles. It is crucial to avoid the spread of bacteria and viruses. Since the porch's cleaning and disinfection function is very important, the porch's new function design is beneficial to the family's healthy development, and the accumulated behavior habits will become a natural health consciousness. Of course, there are many different types of indoor spaces in a house. Only a few types may be represented by the three forms in the article. They are not mentioned one by one due to space constraints. In the future, as society develops, there will be more demands. For people's healthy lifestyles, the author believes that interior designs will continue to improve and keep pace with the trends.

\section{Disclosure statement}

The author declares no conflict of interest.

\section{References}

[1] Fu Y, 2006, Research on Spatial Environment of Healthy Housing. Chongqing University, (01).

[2] Zhao F, 2011, Design and Research of Residential Entrance Furniture. Nanjing Forestry University.

[3] 2020, Novel Coronavirus Diagnosis and Treatment Protocol for Pneumonia (Trial Version 5). Jiangsu Journal of Traditional Chinese Medicine, 52(02): 96+95.

[4] Tong H, Huag C, 2015, The Change of Family Structure in Contemporary China and its Social Impact. Northwest Population Journal. (6): 81-84,88.

[5] Liu J, Li Z, 2018, Research on the Design Method of Residential Entrance Space based on Functional Requirements. Architecture and Culture. (05).

[6] Liu Y, 2019, Research on the Design of Entrance Storage Furniture. Nanjing Forestry University.

\section{Publisher's note}

Bio-Byword Scientific Publishing remains neutral with regard to jurisdictional claims in published maps and institutional affiliations. 